\title{
GPU-implemented Tracking in a COMET Drift Chamber
}

\section{Beomki Yeo*, Yannis K. Semertzidis}

Department of Physics, KAIST, Daejeon 34141, Republic of Korea

Center for Axion and Precision Physics Research, IBS, Daejeon 34051, Republic of Korea

E-mail: byeo@kaist.ac.kr, yannis@kaist.ac.kr

\section{Myeong Jae Lee}

Center for Axion and Precision Physics Research, IBS, Daejeon 34051, Republic of Korea

E-mail: myeongjaeleedibs.re.kr

\section{Yoshitaka Kuno}

Department of Physics, Graduate School of Science, Osaka University, Toyonaka, Osaka 560-0043, Japan

E-mail: kuno@phys.sci.osaka-u.ac.jp

\begin{abstract}
Track finding with GPU-implemented fourth order Runge-Kutta (RK) method is investigated to track electrons from muon decay in the COMET drift chamber. In the COMET drift chamber, about 30-40\% of signal events are composed of multiple turns where the right hit assignments to each turn partition are significant in the track finding. Scanning all possible track seeds can resolve the hit-to-track assignment problem with a high robustness about the noise hits, but requires a huge computational cost because of two reasons: 1) The adaptive RK method to propagate the electron track needs small global errors, corresponding to small step sizes. 2) Initial track seeds $\left(\theta, z, p_{x}, p_{y}, p_{z}\right)$ have broad uncertainties, so many initial seeds should be tried and compared. In this presentation, these problems of massive computations are mitigated with 1) the parallel computing of RK track propagation, which assigns each track to each GPU block unit, 2) a better initial guess on the track seeds using the Hough transform and the geometrical property of the cylindrical drift chamber.
\end{abstract}

ICHEP 2018, International Conference on High Energy Physics

4-11 July 2018

Seoul, Republic of Korea

\footnotetext{
* Speaker.
} 
The COMET (COherent Muon to Electron Transition) experiment in J-PARC searches for $\mu-e$ conversion with the sensitivity of $O\left(10^{-15}\right)$ and $O\left(10^{-17}\right)$ in the Phase- 1 and Phase-2, respectively. In Phase-1, where the cylindrical drift chamber is installed, the signal of $\mu-e$ conversion is the mono-energetic electron, and its energy is almost same with the muon mass. Because there is intrinsic backgrounds from DIO (decay-in-orbit) electron whose endpoint energy is similar with that of the signal electron, a sophisticated tracking analysis is required for a high momentum resolution. Tracking in COMET drift chamber is challenged by the multiple turns events composing of about $30-40 \%$ of all analyzable events.

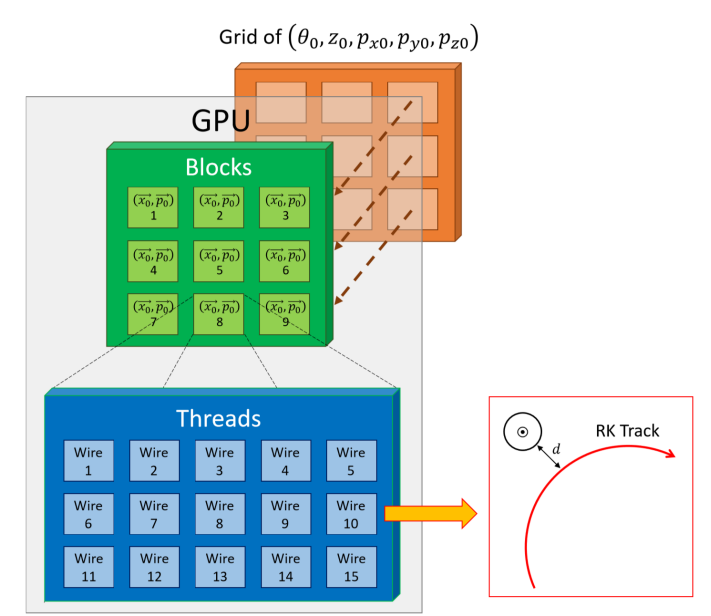

Figure 1: GPU implementation scheme

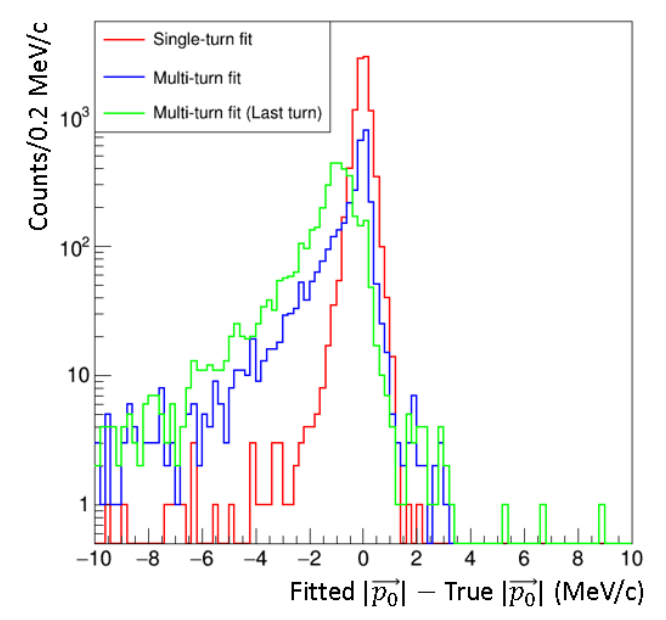

Figure 2: Momentum residual distribution

Tracking for the multiple turn events is done in the following way: 1) Preparing rough track seeds $\left(\theta, z, p_{x}, p_{y}, p_{z}\right)$ using the Hough-transform and geometrical property of the cylindrical drift chamber, and 2) Parallelized GPU-computation of distances between the hits (fired wires) and Runge-Kutta tracks to find the best track seed. The comparison of track seeds is done by comparing the summation of squared distances $(d)$, namely $E=\sum_{\text {wire }} \min \left(d^{2}, \lambda\right)$, where $\lambda$ is the cut-off value to minimize the contribution of hits from non-targeted turns. For the parallelized computation, the analysis of each track seed is independently done in the each block unit of the GPU, and the GPU threads in each block calculate the distances (see Fig. 1). Once the best track seed is found, hits whose distance to the track is less than $\lambda$, are regarded to belong to the targeted turn. Then those hits are fitted using the Kalman filtering to obtain the accurate momentum. The fitted momentum distribution of multiple turn events showed reasonable resolution compared to that of single turn events (see Fig. 2).

\section{Acknowledgement}

This work was supported by IBS-R017-D1 of the Republic of Korea. 